\section{P3.088 HUMAN IMMUNO DEFICIENCY SYNDROME: A GLOBAL CRY}

doi:10.1136/sextrans-2013-051184.0547

'R N M Mbugua, ${ }^{2} \mathrm{E}$ A Bukusi, ${ }^{3} \mathrm{~A}$ wagura. ${ }^{1}$ Kenya Medical Research Institute/Kenya Network of Women Living with HIVIAIDS/Kenyatta National Hospital, Nairobi, Kenya ${ }^{2}$ Kenya Medical Research Institute, Nairobi, Kenya; ${ }^{3}$ Kenya Network of Women Living with HIVIAIDS, Nairobi, Kenya

Objectives The Human Immunodeficiency Virus (HIV) is a retrovirus that attacks the immune system of the host individual, slowly invading and killing T-cells. As the disease progresses, individuals become increasingly susceptible to other illnesses. Eventually usually within 7 to 10 years the compromised immune system will lead to death through another proximate cause. An individual is said to have (AIDS) once their immune system has been severely compromised. Methods The most common channels of transmission are sexual; the other major type of transmission is vertical from mother to child either in the womb, during birth, or while breastfeeding. HIV can also be spread through sharing needles (either by intravenous drug users, or poor hygiene in hospitals) and through transfusions with infected blood.

Results The efficiency of these transmission mechanisms varies. Infection rates are higher for anal than vaginal sex, higher still for mother-to-child transmission, and extremely high (close 100 percent) for transfusion with infected blood.

Drugs that dramatically slow the progression of HIV have become available in recent years. Use of these regimens in the developing world is rare, due both to the cost of the drugs (even in generic form) and the difficulty of administering daily drug cocktails on a continent with few doctors.

Conclusions Interventions in Africa have focused more on prevention and treatment of opportunistic infections, including prevention of mother-to-child transmission, education about changes in sexual behaviour, treatment of other sexually transmitted infections (STIs) and treatment of tuberculosis and other disease associated with HIV/AIDS

\section{P3.089 PERINATAL MORBIDITY ASSOCIATED WITH TRICHOMONAS VAGINALIS: A META-ANALYSIS}

doi:10.1136/sextrans-2013-051184.0548

'B Silver, ${ }^{1,2} \mathrm{~A}$ Rumbold, ${ }^{3} \mathrm{M}$ Jamil, ${ }^{3} \mathrm{~J}$ Kaldor, ${ }^{3} \mathrm{R}$ Guy. ${ }^{1}$ Menzies School of Health Research, Alice Springs, Australia; ${ }^{2}$ University of Adelaide, Adelaide, Australia; ${ }^{3} T h e$ Kirby Institute, UNSW, Sydney, Australia

Background Trichomonas vaginalis is common worldwide, with high rates in women of reproductive age. The evidence-base on potential consequences of infection in pregnancy is mixed. We conducted a meta-analysis of studies which examined the association between T.vaginalis infection and adverse pregnancy outcomes.

Methods Following the PRISMA guidelines and MOOSE criteria, we searched the electronic databases Medline, EMBASE and BioMedCentral for articles published from January 1950 to February 2013; reference lists of published studies and reviews were also examined to locate additional eligible studies. The inclusion criteria were studies that assessed the statistical association between T.vaginalis in women and adverse pregnancy outcomes. The primary outcomes of interest were preterm birth, low birth weight and premature rupture of membranes. Meta-analysis methods were used to calculate a summary odds ratio for each outcome.

Results A total of 174 papers were identified, of these 48 full text papers were reviewed and 10 studies met the inclusion criteria. Six studies were cohorts, one a randomised control trial, one a case control study, one a population data linkage study and one a secondary analysis of combined data from four studies. The study populations, outcomes and study quality varied. The summary odds ratios (OR) was 1.4 (95\% CI: 1.3-1.6, p = 0.0) for preterm birth (8 studies; $\mathrm{n}=78,573)$ with negligible heterogeneity between studies (Isq. $=0.0 \%, p=0.44$ ). For low birth weight (3 studies; $\mathrm{n}=14,660)$ the summary OR was 1.6 (95\% CI: $1.1-2.2, \mathrm{p}=0.009)$ with lowmoderate heterogeneity between studies (Isq. $=35.9 \%, p=0.21$ ) and the summary OR was 2.3 (95\% CI: 0.9-6.2, p = 0.09) for premature rupture of membranes (4 studies, $n=14,715$ ) with high heterogeneity (Isq. $=80.0 \%, p=0.002$ )

Conclusion This review suggests that there is an association between T.vaginalis in pregnancy and preterm birth and low birth weight. These findings confirm the importance of screening for the infection, particularly in women of reproductive age in high prevalence areas.

\section{P3.090 BACTERIAL VAGINOSIS AND THE RISK OF TRICHOMONAS VAGINALIS ACQUISITION AMONG HIV-1 NEGATIVE WOMEN}

doi:10.1136/sextrans-2013-051184.0549

1,2 J E Balkus, ${ }^{1,2} \mathrm{~B}$ A Richardson, ${ }^{3} \mathrm{~L} K$ Rabe, ${ }^{4} \mathrm{~T}$ Taha, ${ }^{5} \mathrm{~N}$ Mgodi, ${ }^{6} \mathrm{M} P$ Kasaro, ${ }^{7} \mathrm{~L}$ A Maslankowski, ${ }^{8} \mathrm{G}$ Ramjee, ${ }^{9} \mathrm{~F}$ Hoffman, ${ }^{10,11} \mathrm{~S}$ S Abdool Karim. ${ }^{1}$ Fred Hutchinson Cancer Research Center, Seattle, WA, United States; ' University of Washington, Seattle, WA, United States; ${ }^{3}$ Magee Women's Research Institute, Pittsburgh, PA, United States; ${ }^{4}$ Johns Hopkins School of Public Health, Baltimore, MD, United States; ${ }^{5}$ University of Zimbabwe, Harare, Zimbabwe; ${ }^{6}$ Center for Infectious Disease Research in Zambia, Lusaka, Zambia; ' University of Pennsylvania, Philadelphia, PA, United States; ${ }^{8}$ South

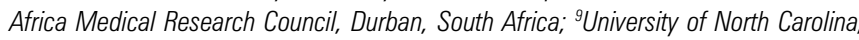
Chapel Hill, NC, United States; ${ }^{10}$ University of KwaZulu-Natal, Durban, South Africa; ${ }^{11}$ Columbia University, New York, NY, United States

Background The vaginal microbiota may play a role in mediating susceptibility to sexually transmitted infections, including Trichomonas vaginalis (TV). This analysis evaluated the association between bacterial vaginosis (BV) and incident TV among women enrolled in a biomedical HIV prevention trial

Methods Data were analysed from HIV-1 seronegative women participating in HIV Prevention Trials Network Protocol 035. At quarterly visits for up to 30 months, participants completed structured interviews and specimens were collected for genital tract infection testing. TV was detected by saline microscopy. BV was characterised by Gram stain using the Nugent score (BV $=7-10$ intermediate $=4-6$; normal $=0-3$ [reference group]). Cox proportional hazards models stratified by study site were used to assess the association between BV at the prior quarterly visit and TV acquisition. Participants were censored at their first TV infection or if they became pregnant or HIV-infected.

Results This secondary analysis included 2,804 participants from Malawi, South Africa, USA, Zambia and Zimbabwe who contributed 13,977 follow-up visits. BV was detected at 5,184 (37.1\%) visits and TV was detected at 352 (2.5\%) visits. After adjusting for age, marital status, hormonal contraceptive use, sexual activity and TV at baseline, intermediate microbiota and BV at the prior visit were independently associated with an increased risk of TV (intermediate microbiota: adjusted hazard ratio $[\mathrm{aHR}]=1.74,95 \%$ confidence inter val [CI] 1.22, 2.47; BV: aHR =3.25, 95\% CI 2.53-4.17). TV at baseline was also associated with an increased risk of TV $(\mathrm{aHR}=2.54 ; 95 \% \mathrm{CI}$ 1.91, 3.36). Sensitivity analyses excluding 202 women with baseline TV showed similar results (BV: aHR $=3.18 ; 95 \%$ CI $2.42-2.19$ ).

Conclusions Women with a Nugent score $>3$ were at an increased risk of acquiring TV. If this relationship is causal, interventions that decrease the incidence of BV and promote a normal vaginal microbiota could potentially contribute to reductions in TV incidence.

\section{P3.091 DISTRIBUTION AND RISK FACTORS OF TRICHOMONAS VAGINALIS INFECTION IN ENGLAND: AN EPIDEMIOLOGICAL STUDY USING ELECTRONIC HEALTH RECORDS FROM SEXUALLY TRANSMITTED INFECTION CLINICS}

doi:10.1136/sextrans-2013-051184.0550 